\title{
INTERACTION REGION LOCAL CORRECTION FOR THE LARGE HADRON COLLIDER*
}

\begin{tabular}{|c|c|c|}
\hline Abstract & Table 1: LHC parameters $\mathrm{f}$ & or protons at collision $(7 \mathrm{TeV})$ \\
\hline $\begin{array}{l}\text { The performance of the Large Hadron Collider (LHC) at } \\
\text { collision energy is limited by the field quality of the inter- } \\
\text { action region (IR) quadrupoles and dipoles. In this paper } \\
\text { we study the impact of the expected field errors of these } \\
\text { magnets on the dynamic aperture (DA). Since the betatron }\end{array}$ & $\begin{array}{l}\text { tunes } \mathrm{H} / \mathrm{V} / \mathrm{L} \\
\beta^{*} \mathrm{IP} 1,5,2,8 \mathrm{H} / \mathrm{V}[\mathrm{m}] \\
\Phi / 2 \mathrm{IP} 1,5,2,8 \mathrm{H} / \mathrm{V}[\mu \mathrm{rad}] \\
\max \mathrm{rms} \text { beam size }[\mathrm{mm}] \\
\max \text { orbit offset } \mathrm{H} / \mathrm{V}[\mathrm{mm}]\end{array}$ & $\begin{array}{l}63.31 / 59.32 / 0.00212 \\
0.5 / 0.5,0.5 / 0.5,15 / 10,13 / 15 \\
0 / 150,150 / 0,0 /-150,0 /-150 \\
1.5 \\
\pm 7.3 / \pm 7.3\end{array}$ \\
\hline
\end{tabular}

phase advance is well defined for magnets that are located in regions of large beta functions, local corrections can be very effective and robust. We compare possible compensation schemes and propose a corrector layout to meet the required DA performance.

\section{INTRODUCTION}

The LHC interaction region consists of a low- $\beta^{*}$ quadrupole triplet (Q1-Q3) and a separation dipole (D1) on either side of the interaction point (IP), Fig. 1. The superconducting triplet quadrupoles are built by FNAL and KEK, and assembled in cryostats at FNAL. The separation dipoles in the high luminosity interactions points IP1 (ATLAS) and IP5 (CMS) are room-temperature magnets supplied by IPN-Novosibirsk. In IP2 (ALICE) and IP8 (LHCB), where the beams are also injected into the two rings, the D1 is a superconducting magnet built by BNL. This magnet has the same coil design as the RHIC arc dipoles, and its field quality is well established. The field quality of the warm D1 is expected to be satisfactory.

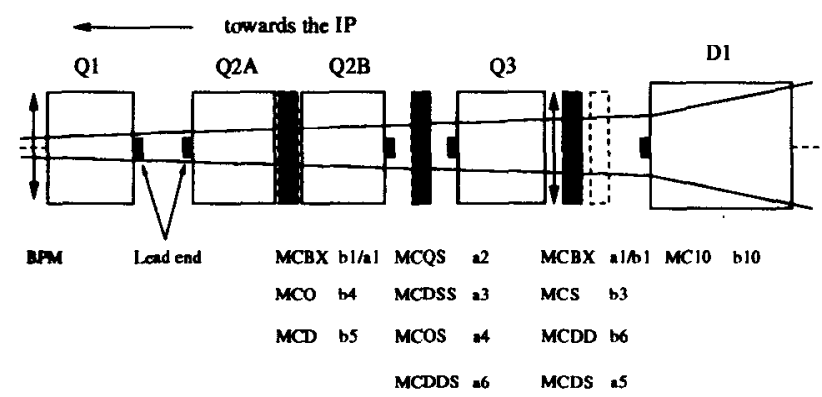

Figure 1: Schematic layout of the LHC inner triplet region.

The target DA for magnet field quality is set at 12 times the transverse rms beam size $\left(12 \sigma_{x y}\right)$ for both injection and collision. During injection and ramping, the impact of IR magnet is small compared with that of the arc magnets. On the other hand, during p-p collision (Tab. 1) the reduction of beam size at IP1 and 5 results in a large beam size $\left(\sigma_{x y}=1.5 \mathrm{~mm}\right)$ at the corresponding triplets. Furthermore, beam-beam interaction requires a crossing angle of $\pm 150 \mu \mathrm{r}$ corresponding to a closed orbit up to $\pm 7.3 \mathrm{~mm}$. The target $12 \sigma_{x y}$ thus corresponds to about $71 \%$ of mag-

\footnotetext{
- Work performed under the auspices of the US Department of Energy.

† Email: weil @bnl.gov
}

net coil radius. Similarly, during ion collision [1] when the beam size is squeezed at IP2, the impact from cold D1 is also noticeable. Compensation of field errors of these cold IR magnets is of primary importance in improving the performance of the LHC at collision [2].

The leading source of DA reduction are the field errors of the FNAL and KEK triplet quadrupoles. The expected errors of the FNAL quadrupole (ver. 2.0) are given in Tab. 2. With the experience of model construction and measurements, and design iterations that occurred through close interaction between the magnet and accelerator physics groups, knowledge and confidence in the expected body and end-field errors has substantially improved. The KEK quadrupole errors are shown in Tab. 3; the coil crosssection of this quadrupole has been recently redesigned in order to substantially reduce the geometric $b_{10}$ error.

In Section 2, we evaluate the DA under nominal collision conditions and explore the optimum quadrupole arrangement to minimize the error impact. In Sections 3 and 4, we review the local IR correction principle and propose a corrector layout.

Table 2: Expected field errors of FNAL low- $\beta^{*}$ quadrupole at collision (version $2.0, R_{\text {ref }}=17 \mathrm{~mm}$ ). $\langle\rangle,. d($.$) and \sigma($. denote the mean, mean uncertainty and rms of the harmonics, respectively.

\begin{tabular}{lcccccc}
\hline$n$ & \multicolumn{3}{c}{ Normal } & \multicolumn{3}{c}{ Skew } \\
& $\left\langle b_{n}\right\rangle$ & $d\left(b_{n}\right)$ & $\sigma\left(b_{n}\right)$ & $\left\langle a_{n}\right\rangle$ & $d\left(a_{n}\right)$ & $\sigma\left(a_{n}\right)$ \\
\hline body & {$[$ unit] } & & & & & \\
3 & - & 0.3 & 0.8 & - & 0.3 & 0.8 \\
4 & - & 0.2 & 0.8 & - & 0.2 & 0.8 \\
5 & - & 0.2 & 0.3 & - & 0.2 & 0.3 \\
6 & - & 0.6 & 0.6 & - & 0.05 & 0.1 \\
7 & - & 0.05 & 0.06 & - & 0.04 & 0.06 \\
8 & - & 0.03 & 0.05 & - & 0.03 & 0.04 \\
9 & - & 0.02 & 0.03 & - & 0.02 & 0.02 \\
10 & - & 0.02 & 0.03 & - & 0.02 & 0.03 \\
LE & {$[$ unit-m] } & \multicolumn{7}{c}{$($ length=0.41 m) } \\
2 & - & - & - & 16.4 & - & - \\
6 & 0.82 & 0.82 & 0.31 & - & 0.21 & 0.06 \\
10 & -0.08 & 0.08 & 0.04 & - & 0.04 & 0.04 \\
RE & {$[$ unit.m] } & \multicolumn{7}{c}{$($ length=0.33 m) } \\
6 & - & 0.41 & 0.31 & - & - & - \\
10 & -0.08 & 0.08 & 0.04 & - & - & - \\
\hline
\end{tabular}


Table 3: Expected field errors of KEK low- $\beta^{*}$ quadrupole at collision (version 2.0, $R_{\text {ref }}=17 \mathrm{~mm}$ ).

\begin{tabular}{lcccccc}
\hline$n$ & \multicolumn{3}{c}{ Normal } & \multicolumn{3}{c}{ Skew } \\
& $\left\langle b_{n}\right\rangle$ & $d\left(b_{n}\right)$ & $\sigma\left(b_{n}\right)$ & $\left\langle a_{n}\right\rangle$ & $d\left(a_{n}\right)$ & $\sigma\left(a_{n}\right)$ \\
\hline body & [unit] & & & & & \\
3 & - & 0.51 & 1.0 & - & 0.51 & 1.0 \\
4 & - & 0.29 & 0.57 & - & 0.29 & 0.57 \\
5 & - & 0.19 & 0.38 & - & 0.19 & 0.38 \\
6 & - & 0.5 & 0.19 & - & 0.10 & 0.19 \\
7 & - & 0.05 & 0.06 & - & 0.05 & 0.06 \\
8 & - & 0.02 & 0.03 & - & 0.02 & 0.03 \\
9 & - & 0.01 & 0.01 & - & 0.01 & 0.01 \\
10 & 0.25 & 0.03 & 0.01 & - & 0.01 & 0.01 \\
LE & {$[$ unit.m] } & & (length=0.45 m) & & \\
2 & - & - & - & 13.4 & - & - \\
6 & 2.28 & - & - & 0.07 & - & - \\
10 & -0.17 & - & - & -0.02 & - & - \\
\hline
\end{tabular}

\section{DA TRACKING ANALYSIS}

The leading errors of the IR quadrupoles are the systematic $b_{6}$ and $b_{10}$, which are allowed by the quadrupole symmetry. We assess the effect of magnetic errors by the tune spread of particles with amplitudes of up to 6 times the transverse rms beam size $\left(6 \sigma_{x y}\right)$, and by the DA determined by $6 \mathrm{D}$ TEAPOT [3] tracking after either $10^{3}$ or $10^{5}$ turns, averaged over 10 random sets of magnetic errors at 5 emittance ratios $\epsilon_{x} / \epsilon_{y}$. Tracked particles have 2.5 times the rms momentum deviation $\left(2.5 \sigma_{p}\right)$ [2]. Uncertainties in the mean are set at their full amount with either plus or minus sign.

The tune spread due to multipole errors scales as $\left(x_{c}+\right.$ $\left.\beta_{x y} \epsilon_{x y}\right)^{n / 2} / \epsilon_{x y}$, where $x_{c}$ is the closed orbit, $\beta_{x y}$ the lattice $\beta$-function and $\epsilon_{x y}$ the emittance. The $b_{10}$ error of the KEK magnets alone produces a tune spread of $0.61 \times 10^{-3}$ at $6 \sigma_{x y}$ thereby reducing the DA by $2 \sigma_{x y}$ (Tab. 4).

A possibility for reducing the impact of the KEK geometric $b_{10}$ could be to adopt a "mixed" triplet scheme where Q1 and Q3 are KEK quadrupoles and Q2 FNAL quadrupoles. This arrangement would lead to a $30 \%$ reduction of the tune spread, and an $18 \%$ increase of the DA, as shown in Tab. 4.

The mixed arrangement increases the possibility for magnet sorting $[4,5]$ and helps randomizing the uncertainty. It may also reduce the number of needed spare magnets and simplifies the engineering process. However, combining quadrupoles of different transfer functions implies a more complicated powering scheme. While a common bus is still possible, retaining the natural compensation of ripple in a triplet, dynamic behaviour at injection related to snap back and eddy-current effects need to be verified [6].

In order to estimate the $b_{6}$ impact, we assume that FNAL magnets are placed at IP1 and 5 and gradually decrease the total $b_{6}$ to $30 \%$ of its original value assuming a positive $d\left(b_{6}\right)$. Tab. 4 shows a steady increase of the DA from 9.3 $\sigma_{x y}$ to $12.1 \sigma_{x y}$.

The orientation of the quadrupoles was chosen to minimize the lead end $b_{6}$ impact [2]. With the mixed quadrupole
Table 4: Comparison of DA for various triplet arrangements ( $10^{3}$-turn DA in units of $\sigma_{x y}$ with $1 \sigma_{x y}$ step size).

\begin{tabular}{|c|c|c|c|}
\hline Case & DA mean & DA rms & DA min \\
\hline \multicolumn{4}{|c|}{ FNAL IP5, 8; KEK IPI, 2: } \\
\hline & 8.5 & 1.4 & 7 \\
\hline without $b_{10}$ & 10.3 & 1.5 & 7 \\
\hline \multicolumn{4}{|c|}{ FNAL as Q2; KEK as Q1, Q3 (mixed): } \\
\hline & 10.0 & 1.5 & 8 \\
\hline reversed Q3 LE & 9.6 & 2.0 & 6 \\
\hline \multicolumn{4}{|c|}{ FNAL IPI, 5; KEK IP2, 8: } \\
\hline & 9.3 & 2.1 & 6 \\
\hline $80 \% b_{6}$ & 9.9 & 2.0 & 6 \\
\hline $50 \% b_{6}$ & 11.0 & 1.8 & 8 \\
\hline $30 \% b_{6}$ & 12.1 & 1.7 & 9 \\
\hline
\end{tabular}

scheme, the minimization is less effective however. In order to reduce the number of electric buses through Q3, it was further suggested to reverse the orientation of Q3. This leads to a reduction of the average DA of 0.4 sigma, and to an increase of $b_{6}$ corrector strength. As the random $b_{6}$ is large, this effect could be alleviated by sorting. $[4,5]$

\section{IR COMPENSATION SCHEMES}

\subsection{Two-Element Correction Principle}

The error compensation is based on the minimization of action-angle kicks [2] produced by each multipole error $b_{n}$ (or $a_{n}$ ) over a pair of inner triplets. Using two correction elements of each multipole order $c_{n}$ (either $a_{n}$ or $b_{n}$ ), we minimize the sum

$$
\int_{L} d l C_{z} B_{0} c_{n}+(-)^{n} \int_{R} d l C_{z} B_{0} c_{n}, \quad z=x, y
$$

taking advantage of the negligible betatron phase advance within each triplet and D1, and approximate the phase advance between the triplets by $180^{\circ}$. The integral is over the entire left-hand-side (L) or right-hand-side (R) MQX triplet and D1. In dipoles $B_{0}$ is simply the main field, in quadrupoles it is the field at the reference radius $R_{\text {ref }}$. In general, the weights $C_{z}$ in Eq. 1 are chosen according to the multipoles as:

\begin{tabular}{ccccc}
\hline & even $b_{n}$ & odd $b_{n}$ & even $a_{n}$ & odd $a_{n}$ \\
\hline$C_{x}$ & $\beta_{x}^{n / 2}$ & $\beta_{x}^{n / 2}$ & $\beta_{x}^{(n-1) / 2} \beta_{y}^{1 / 2}$ & $\beta_{x}^{(n-1) / 2} \beta_{y}^{1 / 2}$ \\
$C_{y}$ & $\beta_{y}^{n / 2}$ & $\beta_{x}^{1 / 2} \beta_{y}^{(n-1) / 2}$ & $\beta_{x}^{1 / 2} \beta_{y}^{(n-1) / 2}$ & $\beta_{y}^{n / 2}$ \\
\hline
\end{tabular}

The compensation is equally effective for both intersecting beams, since the optics of the interaction region is antisymmetric. However, it does not take into account the closed-orbit deviation due to the crossing angle, and the fact that the crossing planes are respectively vertical and horizontal in the two high luminosity interaction points. On the other hand, the effect of this closed orbit feeddown is partially compensated by the feeddown from the correctors.

\subsection{Correction Scheme Comparison}

There are three corrector packages (MCX1, MCX2, MCX3) in each triplet, Fig. 1. Each MCX1 and MCX3 contains two dipole layers, and each MCX2 contains 
Table 5: Comparison of local IR corrector effectiveness assuming that IR quadrupole errors are measured to a $5 \% \mathrm{rms}$ accuracy. The DA is given in units of $\sigma_{x y}$. The physical aperture of $60 \mathrm{~mm}$ corresponds to about $14 \sigma_{x y}$.

\begin{tabular}{lccc}
\hline Case & DA mean & DA rms & DA min \\
\hline UNMIXED: & & & \\
no correction & 8.5 & 1.4 & 7 \\
scheme 1 & 11.8 & 2.4 & 8 \\
scheme 2 & 12.1 & 2.2 & 9 \\
scheme 3 & 15.4 & 1.8 & 12 \\
scheme 4 & 15.9 & 1.7 & 13 \\
\hline MDXED: & & & \\
no correction & 10.0 & 1.5 & 8 \\
scheme 1 & 12.8 & 1.1 & 10 \\
scheme 2 & 13.2 & 1.3 & 11 \\
scheme 3 & 16.1 & 1.8 & 13 \\
scheme 4 & 17.6 & 1.6 & 14 \\
\hline
\end{tabular}

scheme 1: $b_{3}, b_{4}, b_{5}, b_{6}, a_{3}, a_{4}, a_{6}$ scheme 2: $b_{3}, b_{4}, b_{5}, b_{6}, a_{3}, a_{4}, a_{5}, a_{6}$ scheme 3: $b_{3}, b_{4}, b_{5}, b_{6}, b_{10}, a_{3}, a_{4}, a_{6}$ scheme 4: $b_{3}, b_{4}, b_{5}, b_{6}, b_{10}, a_{3}, a_{4}, a_{5}, a_{6}$

a skew quadrupole layer. A straightforward approach (scheme 1) is to have 3 additional layers of nonlinear skew multipoles $\left(a_{3}, a_{4}, a_{6}\right)$ for MCX2, and two additional layers of nonlinear multipoles for MCX1 and MCX3. These layers could be a combination of any of $b_{3}, b_{4}, b_{5}$ and $b_{6}$ layers. For each multipole, two correction elements, located symmetrically at both sides of the IP, can be activated to minimize the kick in both the $x$ and $y$ directions (compare Eq. 1). Due to the lattice symmetry both beams are corrected.

Scheme 1 increases the DA by $38 \%$ in the unmixed and $28 \%$ in the mixed case. With an additional $a_{5}$ corrector (scheme 2 ) the improvement is $42 \%$ and $32 \%$ respectively. A further improvement can be achieved using a $b_{10}$ corrector, as shown in Tab. 5 and Fig. 2. We also investigated the effect of misalignment of the corrector layers. With an rms misalignment of $0.5 \mathrm{~mm}$ in the horizontal and vertical planes we find no degradation of the DA.

The required strength of the multipole correctors, Tab. 6,

Table 6: Needed and available corrector strength.

\begin{tabular}{|c|c|c|c|}
\hline \multirow[t]{2}{*}{ Order } & \multirow{2}{*}{$\begin{array}{c}\text { Available } \\
\text { strength } \\
{[\mathrm{T}]}\end{array}$} & \multicolumn{2}{|c|}{ Needed strength } \\
\hline & & $\begin{array}{c}(\text { mean } \pm S D) \\
{[\mathrm{T}]}\end{array}$ & $\begin{array}{c}(\text { mean }+6 S D) \\
{[\mathrm{T}]}\end{array}$ \\
\hline$b_{3}$ & 0.100 & $0.0023 \pm 0.0027$ & 0.018 \\
\hline$b_{4}$ & 0.066 & $0.0057 \pm 0.0043$ & 0.032 \\
\hline$b_{5}$ & 0.037 & $0.0015 \pm 0.0018$ & 0.012 \\
\hline$b_{6}$ & 0.020 & $0.0075 \pm 0.0016$ & 0.017 \\
\hline$b_{10}$ & 0.0030 & $0.0011 \pm 0.0002$ & 0.0023 \\
\hline$a_{3}$ & 0.155 & $0.012 \pm 0.009$ & 0.066 \\
\hline$a_{4}$ & 0.086 & $0.014 \pm 0.008$ & 0.062 \\
\hline$a_{6}$ & 0.020 & $0.0021 \pm 0.0016$ & 0.012 \\
\hline$a_{5}$ & 0.044 & $0.014 \pm 0.008$ & 0.062 \\
\hline
\end{tabular}
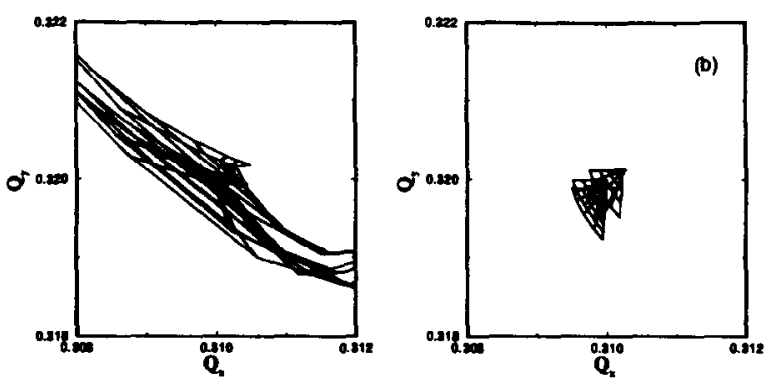

Figure 2: Effect of IR multipole correction on the covered tune space. $(a)$ shows the uncorrected machine and $(b)$ the corrected machine with scheme 4.

can be provided by $50 \mathrm{~cm}$ long spool pieces wound using the LHC sextupole corrector wire and operating at less than $50 \%$ margin at $600 \mathrm{~A}$ [7]. At IP2, the IR correctors are also designed to reduce the effect of the D1 errors during low$\beta^{*}$ heavy ion operations [1]

\subsection{Short versus long term tracking}

Finally we re-confirmed [2] the difference between the DA determined after $10^{3}$ and $10^{5}$ turns for two selected cases, an uncorrected machine and a corrected machine. The difference (Tab. 7) is $0.7 \sigma_{x y}$ or $7 \%$ for the uncorrected case, and $0.9 \sigma_{x y}$ or $5 \%$ for the corrected case.

Table 7: Comparison of 1,000-turn and 100,000-turn DA.

\begin{tabular}{lccc}
\hline Case & DA mean & DA rms & DA min \\
\hline no correction $\left(10^{3}\right)$ & 10.0 & 1.5 & 8 \\
no correction $\left(10^{5}\right)$ & 9.3 & 1.4 & 7 \\
\hline scheme 4 $\left(10^{3}\right)$ & 17.6 & 1.6 & 14 \\
scheme 4 $\left(10^{5}\right)$ & 16.7 & 1.5 & 13 \\
\hline target $\left(10^{5}\right)$ & 12 & - & 10 \\
\hline
\end{tabular}

\section{SUMMARY}

Local nonlinear IR correctors, up to multipole order 6, are proposed for compensating the IR quadrupole errors. These correctors can improve the DA by $2-3 \sigma_{x y}$. Mixing magnets of different origin can help reach the target DA as its improvement is about $1.5 \sigma_{x y}$. This would be equivalent to a reduction of the systematic $b_{10}$ and uncertainty of $b_{6}$ errors of about $50 \%$. Further benefit of mixing could be expected through randomizing the uncertainties and broader selection of the magnets.

We thank J. Gareyte, J.-P. Koutchouk, O. Brining and $J$. Miles for lattice assistance and discussions, and many others, including M. Harrison, A. Ijspeert, J. Kerby, M.J. Lamm, S. Peggs, T. Sen, R. Talman, T. Taylor and A.V. Zlobin.

\section{REFERENCES}

[1] V. Ptitsin, W. Fischer, J. Wei, PAC99 (1999).

[2] J. Wei et al., "US-LHC IR magnet error analysis and compensation", EPAC 1998 proceedings (1998) p. 380.

[3] L. Schachinger, R. Talman, Part. Accel. 22, 35 (1987).

[4] J. Wei, R. Gupta, M. Harrison, A. Jain, et al, PAC99 (1999).

[5] J. Shi, Nuclear Instruments and Methods A (1999).

[6] J-P. Koutchouk, private communications.

[7] M. Karppinen, private communications. 